\title{
Hybrid model of light propagation in random media based on the time-dependent radiative transfer and diffusion equations
}

\author{
Hiroyuki Fujii ${ }^{\mathrm{a}, 1}$, Shinpei Okawa ${ }^{\mathrm{b}}$, Yukio Yamada ${ }^{\mathrm{c}}$, Yoko Hoshi ${ }^{\mathrm{a}}$ \\ ${ }^{a}$ Tokyo Metropolitan Institute of Medical Science, 2-1-6 Kamikitazawa, Setagaya, Tokyo 156-8506 Japan \\ ${ }^{b}$ National Defense Medical College, 3-2 Namiki, Tokorozawa, Saitama 359-8513 Japan \\ ${ }^{c}$ University of Electro-Communications, 1-5-1 Chofugaoka, Chofu, 182-8585 Japan
}

\begin{abstract}
Numerical modeling of light propagation in random media has been an important issue for biomedical imaging, including diffuse optical tomography (DOT). For high resolution DOT, accurate and fast computation of light propagation in biological tissue is desirable. This paper proposes a space-time hybrid model for numerical modeling based on the radiative transfer and diffusion equations (RTE and DE) in random media under refractive-index mismatching. In the proposed model, the RTE and DE regions are separated into space and time by using a crossover length and the time from the ballistic regime to the diffusive regime, $\rho_{D A} \sim 10 / \mu_{t}^{\prime}$ and $t_{D A} \sim 20 / v \mu_{t}^{\prime}$ where $\mu_{t}^{\prime}$ and $v$ represent a reduced transport coefficient and light velocity, respectively. The present model succeeds in describing light propagation accurately and reduces computational load by a quarter compared with full computation of the RTE.
\end{abstract}

Keywords: Radiative transfer equation; Diffusion equation; Hybrid scheme; Discrete ordinate and finite difference method; Light propagation in homogeneous random media.

\section{Introduction}

Diffuse optical tomography (DOT) offers the potential to monitor oxygenation in biological tissue noninvasively $[1,2]$. It requires an algorithm to estimate the distribution of optical properties in tissue from measurements at the boundaries of the medium studied [3]. The algorithm essentially consists of two parts. One is a forward model to calculate the light propagation in a medium and the resultant outward reemissions at the boundary of the medium. The other is an inverse model to search for a distribution of the optical properties by minimizing differences between the calculated and experimental data.

As a forward model, it has been widely accepted that the radiative transfer equation (RTE) provides an accurate description of light propagation phenomena by comparing with experimental data using tissue phantoms [4]. Doe to this, the applicability of the RTE has been investigated to improve DOT images [5]. With its accurate calculations of light properties, the RTE still suffers from the disadvantage of high computation load due to the complexity of the integro-differential equations and high number of independent variables. Thus, most forward models are based on the diffusion equation (DE) [6, 7], which is deduced from the RTE. Use of the DE reduces computational times and memory requirements significantly compared to that of the RTE. However, the DE is known to be invalid in the vicinity of sources and absorbing objects [8]. As a result DE-based

\footnotetext{
Email address: fujii-hr@eng.hokudai.ac.jp (Hiroyuki Fujii)

${ }^{1}$ Now at Hokkaido University, Kita 13, Nishi 8, Kita-ku, Sapporo, Hokkaido, 060-8628, Japan
}

models are susceptible to errors around these sources and objects, leading to low quality DOT images.

Alternative models for the accurate and efficient calculation of light propagation have also been proposed [9, 10, 11, 12, 13, 14], and among these a hybrid model based on the RTE and DE is one of the most promising approaches. Tarvanien et al. have proposed the hybrid model in frequency domain [10, 11, 12], and Gorpas et al. have applied this model to fluorescence imaging $[13,14]$. It has been successfully shown that the light propagation calculated from this model is in good agreement with the RTE calculations. The hybrid model is based on the DE giving accurate results far from sources. In the model, the whole region of a medium is divided in the RTE region at short sourcedetector distances $\left(\rho<\rho_{D A}\right)$ and the DE region at long distances $\left(\rho>\rho_{D A}\right)$, where $\rho_{D A}$ represents a crossover length from the ballistic regime to the diffusive regime [15]. Despite the good results, the estimates of $\rho_{D A}$ involve trial and error, and are medium-dependent. As a result there is still a need for a model where $\rho_{D A}$ is expressed in terms of the optical properties, independently of the media involved. Also, due to high computational loads of solving the time-dependent RTE, only few publications showing the results of the RTE in time-domain are found [16].

This paper extends the concept of the hybrid model in the steady state legitimately to the time domain as the time domain has more information of light propagation than a steady state system. In this extended hybrid model, the RTE and DE regions are divided in space and time by using the crossover length $\rho_{\text {DA }}$ and a crossover time $t_{\mathrm{DA}} \sim \rho_{D A} / \mathrm{v}$. To estimate the $\rho_{\mathrm{DA}}$ and $t_{\mathrm{DA}}$ without reference to media, the time development of light

May 19, 2014 

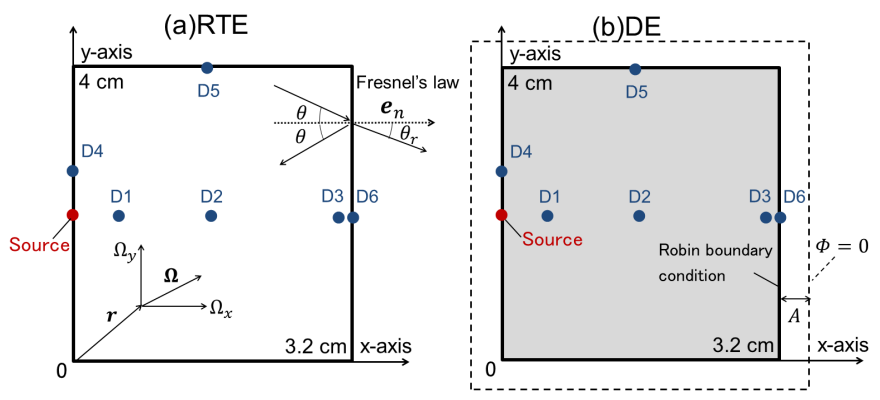

Figure 1: Two-dimensional homogeneous $3.2 \mathrm{~cm} \times 4.0 \mathrm{~cm}$ media for numerical calculations based on (a) the RTE and (b) the DE. A light pulse is injected at a position $(x, y)=(0.0,2.0)$ (in $\mathrm{cm}$ ) represented by the red dots. Six blue dots represent detectors, D1 to D6, at $(x, y)=(0.5,2.0),(2.0,2.0)$, and $(3.1,2.0)$ for $\mathrm{D} 1, \mathrm{D} 2$, and D3 inside the medium, and at $(2.3,0),(2.0,4.0)$, and $(3.2,2.0)$ for D4, D5, and D6 on the boundary. The refractive-index mismatched boundary condition is employed. Fresnel's law is adopted in the RTE, and the Robin boundary condition is adopted in the DE.

propagation based on the RTE and DE are investigated with the refractive-index mismatched boundary condition. The accuracy and computational efficiency of the proposed model is tested by a comparison with numerical results based on the RTE.

This paper is organized as follows. The following section (2) provides a brief explanation of numerical models of the RTE, DE, and the hybrid model proposed here. Section 3 provides numerical results of the light propagation based on the three models under several conditions. Finally, conclusions are detailed in Section 4.

\section{Numerical model}

This paper uses homogeneous $2 \mathrm{D}$ rectangular media $(3.2 \mathrm{~cm}$ $\times 4.0 \mathrm{~cm}$ ) as shown in Fig. 1 throughout.

\subsection{Radiative transfer equation}

Light propagation in random media is described by the intensity $I(\boldsymbol{r}, \boldsymbol{\Omega}, t)$, which is the energy distribution of photons described by the position $\boldsymbol{r}=(x, y)$, angular direction $\boldsymbol{\Omega}=$ $\left(\Omega_{x}, \Omega_{y}\right)$, and time $t$. Considering photon-matter interactions as transit, absorption, and scattering, this makes it possible to derive the RTE [17],

$$
\begin{aligned}
& {\left[\frac{\partial}{v \partial t}+\boldsymbol{\Omega} \cdot \nabla+\mu_{t}(\boldsymbol{r})\right] I(\boldsymbol{r}, \boldsymbol{\Omega}, t) } \\
= & \mu_{S}(\boldsymbol{r}) \int_{S} d \boldsymbol{\Omega}^{\prime} P\left(\boldsymbol{\Omega}, \boldsymbol{\Omega}^{\prime}\right) I\left(\boldsymbol{r}, \boldsymbol{\Omega}^{\prime}, t\right)+q(\boldsymbol{r}, \boldsymbol{\Omega}, t),
\end{aligned}
$$

where $\mu_{t}(\boldsymbol{r})$ is given by the sum of the absorption $\mu_{a}(\boldsymbol{r})$ and scattering coefficients $\mu_{s}(\boldsymbol{r}), v$ is the velocity of light in the target medium, $P\left(\boldsymbol{\Omega}, \boldsymbol{\Omega}^{\prime}\right)$ is a scattering phase function providing a scattering probability from the $\boldsymbol{\Omega}^{\prime}$ before scattering to the $\boldsymbol{\Omega}$ after scattering, and $q(\boldsymbol{r}, \boldsymbol{\Omega}, t)$ is the light source. In the above formulation, polarization and inelastic scattering of photons are disregarded.

Like other studies the present study employs the HenyeyGreenstein phase function for $P\left(\boldsymbol{\Omega}, \mathbf{\Omega}^{\prime}\right)$ due to its simplicity
[18] and for the two dimensional cases, this function is given as

$$
P\left(\boldsymbol{\Omega} \cdot \mathbf{\Omega}^{\prime}\right)=\frac{1}{2 \pi} \frac{1-g^{2}}{1+g^{2}-2 g \boldsymbol{\Omega} \cdot \mathbf{\Omega}^{\prime}},
$$

where the anisotropic parameter $g$ is defined as an expectation value of $\boldsymbol{\Omega} \cdot \boldsymbol{\Omega}^{\prime}$ for the function $P\left(\boldsymbol{\Omega}, \boldsymbol{\Omega}^{\prime}\right)$. In this paper, the value of $g$ is chosen to be zero or positive for modeling isotropic and forward scatterings in biological tissue and phantoms. Due to the elastic scattering, $P$ satisfies the normalized condition $\int d \boldsymbol{\Omega} P\left(\boldsymbol{\Omega} \cdot \mathbf{\Omega}^{\prime}\right)=1$.

The source $q(\boldsymbol{r}, \boldsymbol{\Omega}, t)$ takes the form of a delta function as $\delta\left(\boldsymbol{r}-\boldsymbol{r}_{s}\right) \delta\left(\boldsymbol{\Omega}-\boldsymbol{\Omega}_{s}\right) \delta(t)$, where $\boldsymbol{r}_{s}$ and $\boldsymbol{\Omega}_{s}$ denote the position and the angular direction of an incident pulse, respectively.

At the boundary, the refractive-index mismatching is considered, this is a more realistic boundary condition than the nonreentry boundary condition. The reflection and refraction at the boundary are described by Fresnel's law, which gives the reflectivity $R(n, \theta)$ as

$$
R(n, \theta)= \begin{cases}\frac{1}{2}\left[\frac{\sin ^{2}\left(\theta_{r}-\theta\right)}{\sin ^{2}\left(\theta_{r}+\theta\right)}+\frac{\tan ^{2}\left(\theta_{r}-\theta\right)}{\tan ^{2}\left(\theta_{r}+\theta\right)}\right] & \theta<\theta_{c} \\ 1 & \theta \geq \theta_{c}\end{cases}
$$

where $n$ is the relative refractive index of the medium, $\theta$ is an angle between the outgoing normal vector $\boldsymbol{e}_{n}$ and $\boldsymbol{\Omega}$ as shown in Fig. 1(a), the refraction angle $\theta_{r}=\sin ^{-1}(n \sin \theta)$ is obtained by Snell's law, and $\theta_{c}$ represents the critical angle. The transmissivity $T(n, \theta)$ is given by $1-R(n, \theta)$.

To obtain numerical solutions of the RTE, this equation is replaced by a set of linear equations by using the upwind-discrete ordinate and finite-difference scheme [4]. In this scheme, $I(\boldsymbol{r}, \boldsymbol{\Omega}, t)$ is discretized as $I_{i, j, k, m}$ where $i, j, k$, and $m$ denote the indices of the discrete spatial $\left(x_{i}, y_{j}\right)$, angular $\boldsymbol{\Omega}_{k}$, and temporal $t_{m}$ variables. The integration term in the equation is calculated based on the extended trapezoidal rule, $\int d \mathbf{\Omega} P I \sim \sum_{k} w_{k} P_{k k^{\prime}} I_{k}$ with a weight factor $w_{k}$ and angular index $k$. Commonly, $w_{k}$ is given by $2 \pi / N_{\theta}$ with the number of discrete angles $N_{\theta}$. However, when $N_{\theta}$ is not sufficiently large, normalization of $P$ is not possible, i. e. when $\sum_{k} w_{k} P_{k k^{\prime}} \neq 1$ especially at large values of $g$. For numerical calculations to converge, a modified weight factor is adopted, $w_{k}^{(\text {mod })}=w_{k}\left(\sum_{l} w_{l} P_{l k}\right)^{-1}$ [19] in this study. The temporal derivative is approximated by the forward Euler scheme [16].

\subsection{Diffusion approximation}

In the diffusion approximation (DA), a first order approximation of $I(\boldsymbol{r}, \boldsymbol{\Omega}, t)$ with respect to $\boldsymbol{\Omega}$ and Fick's law is assumed. Then, the diffusion equation (DE) can be derived from the RTE,

$$
\left[\frac{\partial}{v \partial t}-D \nabla^{2}+\mu_{a}\right] \Phi(\boldsymbol{r}, t)=q_{D E}(\boldsymbol{r}, t),
$$

where the fluence rate $\Phi(\boldsymbol{r}, t)$ is given by $\int d \Omega I(\boldsymbol{r}, \boldsymbol{\Omega}, t)$, the diffusion coefficient $D$ is by $\left.\left[2(1-g) \mu_{s}\right)\right]^{-1}$ for the time domain system $[20,21]$, and the isotropic source $q_{D E}(\boldsymbol{r}, t)$ is given by $\int d \Omega q(\boldsymbol{r}, \boldsymbol{\Omega}, t)$. The refractive-index mismatched boundary 


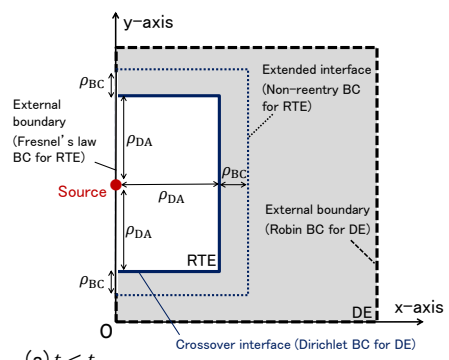

(a) $t<t_{\mathrm{DA}}$

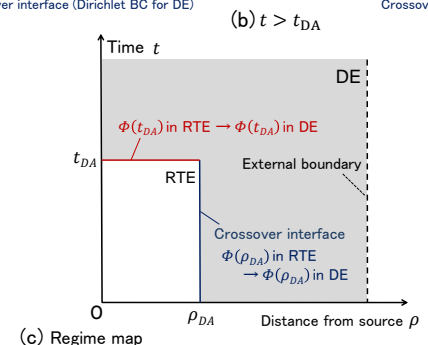

Figure 2: Space-time hybrid model based on the RTE and DE, showing spatial hybrid models for the time period of (a) $t<t_{D A}$ and (b) $t>t_{D A}$, and (c)conceptual regime map. In the spatial hybrid model (a) for $t<t_{D A}$, the whole medium is divided into the RTE (white) and DE (gray) regions separated by the crossover interface (thick solid lines). As the boundary conditions for the RTE, the Fresnel's law is applied at the external boundary in the $y$-axis (thin solid line), and the non-reentry boundary condition $\left(I\left(\boldsymbol{\Omega} \cdot \boldsymbol{e}_{n}<0\right)=0\right)$ is applied at the extended interface (thin solid line). As the boundary conditions for the DE, the $\Phi$ values calculated by the RTE are given at the crossover interface (the Dirichlet boundary condition), and the Robin boundary condition is applied at the external boundary of the medium (thick dashed lines). In the spatial hybrid model (b) for $t>t_{D A}$, the RTE region shrinks and occupies only the $y$-axis. The conceptual regime map of the space-time hybrid model (c) in the plane of time, $t$, versus distance from the source, $\rho$, schematically indicates the RTE (white) and DE (gray) regions which are divided by the crossover length $\left(\rho_{D A}\right)$ and time $\left(t_{D A}\right)$.

condition is reduced to the Robin boundary condition within the DA [22]. For two dimensional cases, it is

$$
\Phi\left(\boldsymbol{r}_{b}, t\right)+\frac{\pi}{2} A(n) D \boldsymbol{e}_{n} \cdot \nabla \Phi\left(\boldsymbol{r}_{b}, t\right)=0,
$$

where the coefficient $A(n) \geq 1$ is given by $\left(1+R_{2}(n)\right) /\left(1-R_{1}(n)\right)$ related to the extrapolated length (see. Fig. 1(b)), and

$$
\begin{aligned}
& R_{1}(n)=\frac{1}{2} \int_{-\pi / 2}^{\pi / 2} d \theta R(\theta, n) \cos \theta \\
& R_{2}(n)=\frac{2}{\pi} \int_{-\pi / 2}^{\pi / 2} d \theta R(\theta, n) \cos ^{2} \theta .
\end{aligned}
$$

In the paper here, the value of $A(n)$ is evaluated (assigned) to the numerical solutions to Eqs. (6) and (3).

Numerical computations of the DE are based on the central spatial finite difference scheme and the temporal forward Euler scheme in time.

\subsection{Hybrid model with space and time separated}

Figure 2 illustrates the space-time hybrid model based on the RTE and DE. The spatial hybrid models are shown in Fig. 2(a) and (b) for the time period of $t<t_{D A}$ and $t>t_{D A}$, respectively. For the time period of $t<t_{D A}$ in Fig. 2(a), the spatial domain of the medium is divided into the RTE (white) and DE (gray) regions by the interface (thick solid lines, called the crossover interface) which is determined by use of the crossover length $\rho_{D A}$ beyond which the DA is valid. The RTE region is a rectangle with a size of $2 \rho_{D A} \times \rho_{D A}$, and the DE region is the rest of the medium.

As the boundary conditions for the RTE, the Fresnel's law is applied at the external boundary in the $y$-axis (thin solid line), and the non-reentry boundary condition $\left(I\left(\boldsymbol{\Omega} \cdot \boldsymbol{e}_{n}<0\right)=0\right)$ is applied at the extended interface (thin dotted lines) which is located in the $\mathrm{DE}$ region by the distance of $\rho_{B C}$ from the crossover interface. The RTE is solved in the extended region with the size of $2\left(\rho_{D A}+\rho_{B C}\right) \times\left(\rho_{D A}+\rho_{B C}\right)$. As the boundary conditions for the DE, the $\Phi$ values at the crossover interface, which are calculated by the RTE, are used as the $\Phi$ values for the DE at the crossover interface (the Dirichlet boundary condition), and at the external boundary of the medium (thick dashed lines) the refractive-index mismatched boundary condition (the Robin boundary condition, Eq. (5)) is used.

The reason of introducing the extended interface where the non-reentry boundary condition is applied for the RTE is as follows. If the non-reentry boundary condition is applied just at the crossover interface (thick solid lines), the calculated $\Phi$ values at the interface are very different from those calculated by the RTE which is solved over the whole medium with the Fresnel's law applied at the whole external boundary. Then, if the $\Phi$ values calculated with the non-reentry boundary condition applied at the crossover interface are used as the Dirichlet boundary condition for the DE, the resultant solutions of the DE will be very different from the true ones. In order to avoid this problem, the non-reentry boundary condition is applied at the extended interface. And the distance, $\rho_{B C}$, is chosen so that the $\Phi$ values at the crossover interface, which are calculated by the RTE in the extended region, are almost the same as those calculated by the RTE which is solved over the whole medium. In other words, $\rho_{B C}$ is chosen as the shortest distance where the $\Phi$ values at the original interface do not change by moving the position of the extended interface further. From a preliminary study, $\rho_{B C}$ is estimated as $3 / \mu_{t}^{\prime}$, where $\mu_{t}^{\prime}=(1-g) \mu_{s}+\mu_{a}$ is the reduced transport coefficient. A work investigating light propagation using the RTE [23] has also concluded that light intensities at positions $3 / \mu_{t}^{\prime}$ or more inside from the external boundary are almost the same as those for an infinite media without the external boundary. So it is reasonable to estimate $\rho_{B C}$ as $3 / \mu_{t}^{\prime}$.

In addition to the spatial hybrid model, temporal hybrid model is constructed, and Fig. 2(c) shows a conceptual regime map of the space-time hybrid model in the plane of time, $t$, versus distance from the source, $\rho$. It schematically indicates the RTE (white) and DE (gray) regions which are divided by the crossover length $\left(\rho_{D A}\right)$ and time $\left(t_{D A}\right)$. Before time reaches $t_{D A}\left(t<t_{D A}\right)$ the spatial hybrid model shown in Fig. 2(a) is used at each time step in the forward Euler scheme for calculation of time development. After time reaches $t_{D A}\left(t>t_{D A}\right)$, the RTE region in the spatial hybrid model shrinks and occupies only the external boundary in the $y$-axis with the length of $2 \rho_{D A}$ (thick solid line) as shown in Fig. 2(b). The RTE is solved in the extended region enclosed by the $y$-axis and extended interface (thin dotted lines) with the size of $2\left(\rho_{D A}+\rho_{B C}\right) \times \rho_{B C}$. 
Table 1: Optical properties of the medium from tissue phantom data [4]. $\mu_{s}^{\prime}=$ $\mu_{s}(1-g)$ represents the reduced scattering coefficient

\begin{tabular}{cccccc}
\hline & $\mu_{a}[1 / \mathrm{cm}]$ & $\mu_{s}[1 / \mathrm{cm}]$ & $\mu_{s}^{\prime}[1 / \mathrm{cm}]$ & $\mathrm{g}$ & $\mathrm{n}$ \\
\hline RTE & 0.35 & 11.6 & 11.6 & 0.0 & 1.56 \\
& 0.35 & 58.0 & 11.6 & 0.8 & 1.56 \\
DE & 0.35 & & 11.6 & & 1.56 \\
\hline
\end{tabular}

The boundary conditions are the same as those for $t<t_{D A}$, i.e., the Fresnel's law at the external boundary in the $y$-axis and the non-reentry boundary condition at the extended interface. The results of $\Phi$ on the $y$-axis by the RTE are used as the Dirichlet boundary condition for the DE similarly to the case for $t<t_{D A}$.

The magnitudes of $\rho_{D A}$ and $\rho_{B C}$ are estimated as $10 / \mu_{t}^{\prime}$ and $3 / \mu_{t}^{\prime}$, respectively, and the details of the estimation are discussed in subsection 3.6.

\section{Numerical results}

\subsection{Calculation conditions}

As shown in Fig. 1, the source (the red dot) is set at the position $\left(x_{s}, y_{s}\right)=(0.0,2.0)$ (in $\left.\mathrm{cm}\right)$ throughout the paper, and light properties are determined at six positions D1 to D6 (the blue dots). At the detectors inside the medium, D1 to D3, the timeresolved fluence rates $\Phi(\boldsymbol{r}, t)$ are measured, while at the detectors at the surface of the medium D4 to D6, the time-resolved photon currents $J^{+}(\boldsymbol{r}, t)$ defined by Eq. (7) are measured.

$$
J^{+}(\boldsymbol{r}, t)=\left\{\begin{array}{l}
\int_{\boldsymbol{\Omega} \cdot \boldsymbol{e}_{n}>0} d \mathbf{\Omega} T(n, \theta)\left(\boldsymbol{\Omega} \cdot \boldsymbol{e}_{n}\right) I(\boldsymbol{r}, \boldsymbol{\Omega}, t) \\
-D \boldsymbol{e}_{n} \cdot \nabla \Phi(\boldsymbol{r}, t)=\frac{2}{\pi A(n)} \Phi(\boldsymbol{r}, t)
\end{array}\right.
$$

Both the RTE and DE are solved numerically giving the same optical properties, and the same size of space and time steps. The optical properties refer to those of a phantom containing $\mathrm{SiO}_{2}$ particles [4] as listed in Table 1. The space step size $\Delta x$ is $0.008 \mathrm{~cm}$, which is shorter than the transport distance (inverse of $\left.\mu_{t}(\boldsymbol{r})\right)$. Number of the discrete solid angles $N_{\theta}$ is 48 , which adequately suppresses any ray effect. The time step size $\Delta t$ needs to satisfy the Courant-Fredrichs-Lewy condition and is given as $0.01 \mathrm{ps}$.

\subsection{Validation of current numerical schemes}

Before discussing the numerical results for two-dimensional finite media, the validity of the numerical schemes proposed here is compared with analytical solutions in infinite media. Analytical solutions of the DE have been derived for a variety of geometries [24, 26]. Analytical solutions of the RTE have been derived for only a few geometries such as infinite media [25, 27] due to the complexity of the integro-differential equations. To perform numerical calculations for infinite media having no boundaries, the source and detector positions are set sufficiently far from the boundaries which have to be obtained in numerical calculations. In Fig. 3, the numerical results of $\Phi$ compare with the analytical solutions based on the RTE $(g=0.0)$ and DE at the source-detector distance $\rho=1$

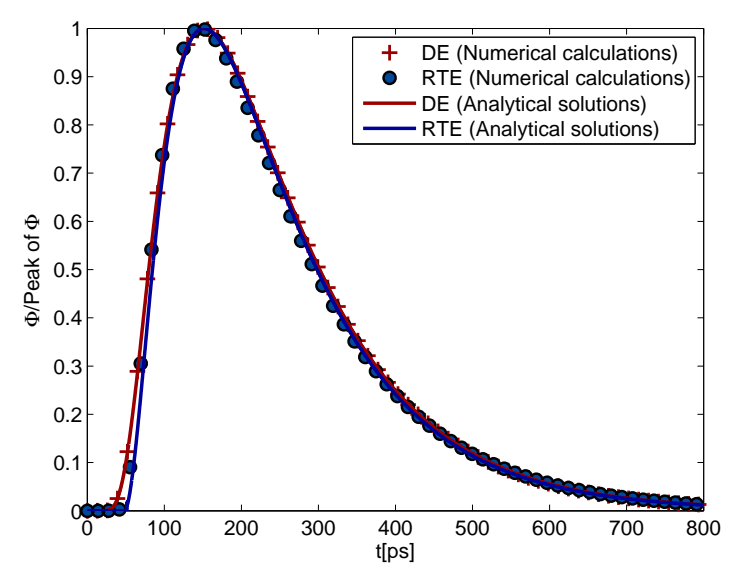

Figure 3: Plot of the fluence rates $\Phi(\rho, t)$ based on the RTE $(g=0.0)$ and the DE at the source-detector distance $\rho=1 \mathrm{~cm}$ in an infinite medium. Red crosses and blue dots represent the numerical results of the DE and the RTE, respectively. Red and blue solid curves denote reported analytical solutions of the DE [24] and RTE [25], respectively.

$\mathrm{cm}$ in an infinite medium. It was confirmed that the numerical results of $\Phi$ are in good agreement with the analytical solutions within the error margins.

\subsection{Light propagation near the source}

In this study, the RTE is solved numerically for four conditions: (1) $g=0.8$ and isotropic source, $(2) g=0.0$ and isotropic source, (3) $g=0.8$ and anisotropic source $\left(\Omega_{s x}, \Omega_{s y}\right)=(1,0)$, and (4) $g=0.0$ and anisotropic source $\left(\Omega_{s x}, \Omega_{s y}\right)=(1,0)$. The DE is solved under only one condition: $D=\left[2(1-g) \mu_{s}\right]^{-1}=$ $0.043 \mathrm{~cm}$ and isotropic source because $D$ is independent of $g$ when $\mu_{s}^{\prime}$ is fixed. Figure 4 shows that time development of $\Phi$ detected at D1 inside the medium for the various conditions, where the source-detector distance $\rho=0.5 \mathrm{~cm}$ is shorter than $\rho_{D A}\left(\rho<\rho_{D A}\right)$. Essentially, photons propagate ballistically and are less scattered in the vicinity of the source position, making the DE invalid in this area. At an early time $t<\rho / v \sim 16 \mathrm{ps}$, no photons arrive at the detector in the RTE-based calculations. In the DE-based calculations, meanwhile, $\Phi$ has nonzero values at this period of time, meaning that the DA fails.

The temporal profiles of $\Phi$ calculated using the RTE are sensitive to the anisotropic factor $g$ and source conditions as shown in Fig. 4. This sensitivity indicates that higher orders of $I$ with respect to $\boldsymbol{\Omega}$ contribute significantly to $\Phi$. Compared with the forward scattering $(g=0.8)$, the temporal profiles of $\Phi$ with the isotropic scattering $(g=0.0)$ tend to behave ballistically, especially at the anisotropic source.

The time development of $J^{+}$at detector D4 $(\rho=0.3 \mathrm{~cm})$ near the source on the boundaries behave similarly to $\Phi$ as shown in Fig. 5. This shows that the RTE-based computations of $J^{+}$are influenced by the values of $g$ and the source conditions. At the detector position, light propagation based on the RTE with $g=$ 0.8 and the anisotropic source show delayed times. This arises as the strong forward scattering and the anisotropic source make backward light propagation late. 


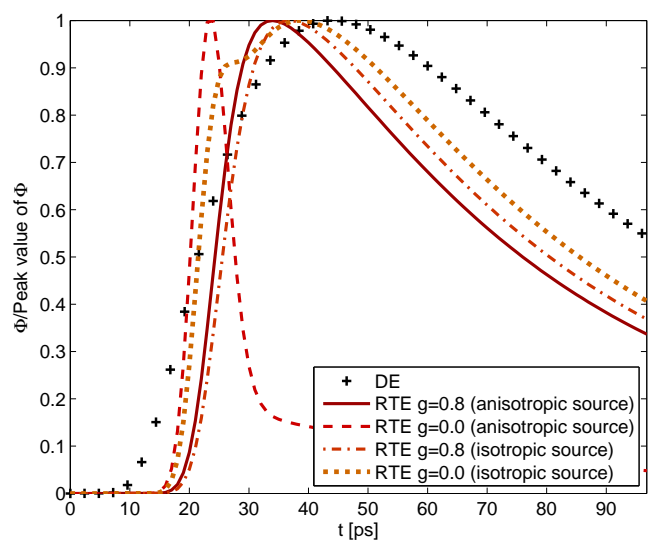

Figure 4: Calculated temporal profiles of the fluence rates $\Phi$ at detector D1 inside the medium with the source-detector distance $\rho=0.5 \mathrm{~cm}$ shorter than $\rho_{D A}$. Crosses represent calculated results of $\Phi$ based on the DE, solid curves those based on the RTE with $g=0.8$ and the anisotropic source $\left(\Omega_{s x}, \Omega_{s y}\right)=$ $(1,0)$, dashed curves those with $g=0.0$ and the anisotropic source $\left(\Omega_{s x}, \Omega_{s y}\right)=$ $(1,0)$, dash-dot (chain) curves with $g=0.8$ and the isotropic source, and dotted curves those with $g=0.0$ and the isotropic source.

On the boundaries, the $J^{+}$calculated using the RTE is qualitatively similar to $\Phi$ and the characteristic times are consistent with both light properties, although the peak value of $J^{+}$is smaller than that of $\Phi$. This is based on a fact that the characteristic times of the intensity $I$ are only weakly dependent on the direction.

\subsection{Light propagation far from the source}

Figures 6 and 7 show the time development of $\Phi$ and $J^{+}$detected at D2, D3, D5, and D6 far from the source. At the long distances $\rho\left(>\rho_{D A}\right)$, the temporal behaviors of $\Phi$ and $J^{+}$are different from those at the short distances $\rho\left(<\rho_{D A}\right)$. The influences of $g$ and the source anisotropy on the light propagation become much smaller, indicating that this data is lost due to multiple scattering. As a result, the temporal profiles based on the RTE at different values of $g$ and source anisotropies are very similar at the detector position. Then, it may be postulated that the DE holds in the region far from the source. However, the numerical results of $\Phi$ obtained from the RTE still have small disagreements with those from the DE. Generally, the times of $\Phi$ and $J^{+}$using the RTE are faster than those using the DE. These disagreements arise in association with the boundary conditions, as will be explained next.

In infinite media, the temporal profiles of $\Phi$ based on the RTE and DE coincide with each other, including the times as shown in Fig. 3. This allows the conclusion that the disagreements in the times in finite media arise from the differences in the boundary conditions of the two equations. When decreasing the values of the coefficient $A$ in Eq. (5), the temporal profiles based on the DE shift towards longer times and approach the profiles based on the RTE. This indicates that the DE holds in the region far from the source, qualitatively. Also, the ratio $t_{\text {ratio }}$ of the time scale based on the RTE to that on the DE is an almost constant value $\sim 1.045$, irrespective of the detector positions.

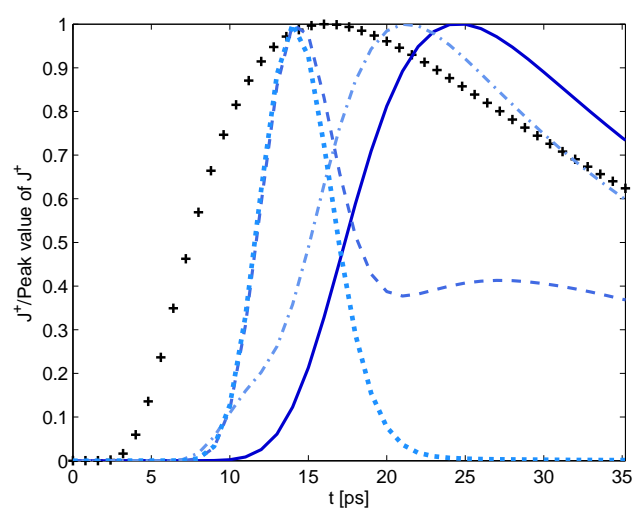

Figure 5: Calculated temporal profiles of the photon currents $J^{+}$at detector D4 on the surface of the medium with a short source-detector distance $\rho=0.3 \mathrm{~cm}$ $\left(<\rho_{D A}\right)$. Crosses represent calculated results based on the DE, solid curves those based on the RTE with $g=0.8$ and the anisotropic source $\left(\Omega_{s x}, \Omega_{s y}\right)=$ $(1,0)$, dashed curves those with $g=0.0$ and the anisotropic source $\left(\Omega_{s x}, \Omega_{s y}\right)=$ $(1,0)$, dash-dot (chain) curves with $g=0.8$ and the isotropic source, and dotted curves those with $g=0.0$ and the isotropic source.

Thus, by time-scaling $t \rightarrow t / t_{\text {ratio }}$, the results of $\Phi\left(t / t_{\text {ratio }}\right)$ based on the DE are consistent with those of $\Phi(t)$ on the RTE, as well as on the changing values of $A$.

\subsection{Spatial distribution of light}

So far, the temporal profiles of light propagations at a fixed position are examined, and the validity of the DA is confirmed at the long distances $\rho\left(>\rho_{D A}\right)$. Then, the spatial distribution of the fluence rate based on the different models is investigated at a fixed time. The fluence rate is calculated by the DE and RTE with $g=0.0$. Figure 8 shows the spatial distributions of $\Phi$ at the short time $t\left(<t_{D A}\right)$. Similarly to the temporal profiles at the short distances in Fig. 4, the spatial distributions at the short time are dependent on the numerical models and conditions. In contrast, at the time longer than $t_{D A}$, the spatial distributions calculated from the DE and RTE are quite similar to each other (Fig. 9). This result indicates that the DA holds at the long time $\mathrm{t}\left(>t_{D A}\right)$.

\subsection{Light propagation based on the hybrid models}

The time resolved profiles of $\Phi$ and $J^{+}$show a single peak as plotted in Figs. 4 and 5, the peak time is dependent on the light scattering by the medium. This suggests that the peak time $t_{\text {peak }}$ may be a fundamental time like that of the mean time of flight. Figure 10 shows a log-log plot of the normalized peak times $t_{\text {peak }} \mu_{t}^{\prime} v$ calculated based on the RTE and DE in the different conditions and as a function of the normalized source-detector distance $\rho \mu_{t}^{\prime}, \mu_{t}^{\prime}=(1-g) \mu_{s}+\mu_{a}$ is the reduced transport coefficient. The normalization is to indicate general features regardless of the optical properties, here, $t_{\text {peak }}$ calculated from the DE is scaled by the $1 / t_{\text {ratio }}$.

At the shorter distances, $\log _{10} \rho \mu_{t}^{\prime} \leq 0.6$, the ballistic mode is dominant in the light propagation, and $t_{\text {peak }}$ is approximately equal to $\sim \rho / v$. At the longer distances, $1.0 \leq \log _{10} \rho \mu_{t}^{\prime}$, the diffusion process is dominant, and the gradients of $\log _{10} t_{\text {peak }}$ 


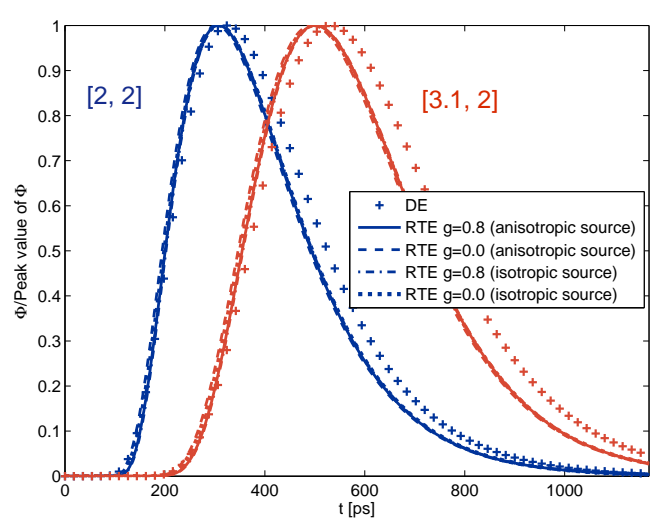

Figure 6: Computed temporal profiles of $\Phi$ at detectors D2 $(\rho=2.0 \mathrm{~cm})$ and $\mathrm{D} 3(\rho=3.1 \mathrm{~cm})$ inside the medium far from the source $\left(\rho>\rho_{D A}\right)$. Crosses represent calculated results based on the DE, solid curves those based on the RTE with $g=0.8$ and the anisotropic source $\left(\Omega_{s x}, \Omega_{s y}\right)=(1,0)$, dashed curves those with $g=0.0$ and the anisotropic source $\left(\Omega_{s x}, \Omega_{s y}\right)=(1,0)$, dash-dot (chain) curves with $g=0.8$ and the isotropic source, and dotted curves those with $g=0.0$ and the isotropic source.

Table 2: Computation times using the three models: RTE, hybrid model, and DE (time integration $1200 \mathrm{ps)}$

\begin{tabular}{lccc}
\hline & RTE & Hybrid & DE \\
\hline Computation time [min] & 2658 & 648 & 20 \\
\hline
\end{tabular}

against $\log _{10} \rho$ for the RTE and DE calculations are in good agreement. This agreement indicates that the boundary conditions have an insignificant effect on the gradients. Around the region $0.6 \leq \log _{10} \rho \mu_{t}^{\prime} \leq 1.0$ there is a crossover which appears ballistic to diffusive motions and here the values of $g$ and source anisotropy contribute strongly to $t_{\text {peak }}$. From Fig. 10, the ballistic regime is defined as the $\log _{10} \rho \mu_{t}^{\prime} \leq 1.0$ region, including the crossover, and the diffusive regime is defined as the $1.0 \leq \log _{10} \rho \mu_{t}^{\prime}$ region.

Figure 10 suggests values of the crossover length $\rho_{D A}$ and time $t_{D A}$ to be about $10 / \mu_{t}^{\prime}$ and $20 / v \mu_{t}^{\prime}$, irrespective of a medium with optical properties. Using $\rho_{D A}$ and $t_{D A}$, it becomes possible to construct a space-time hybrid model based on the RTE and $\mathrm{DE}$ in the way explained in Section 2.3. For the construction of the hybrid model, an additional length $\rho_{B C}$ is necessary and this length characterizes the boundary effects. In a previous previous study [23], $\rho_{B C}$ was estimated to be approximately $3 / \mu_{t}^{\prime}$. In the current paper, the values of $\rho_{D A}, \rho_{B C}$, and $t_{D A}$ are $1.15 \mathrm{~cm}$, $0.4 \mathrm{~cm}$, and $119.1 \mathrm{ps}$, respectively.

To implement the temporal hybrid, quantitative agreement of times for the RTE and DE is required. As discussed in the previous subsection, however, the characteristic times calculated using the DE are different from those using the RTE because of the difference in boundary conditions. A number of methods to achieve coincidence of the times for the RTE and DE appear possible. In this paper, the $A$ value of the Eq. (5) is changed and reset to one that enables qualitative agreements of the light propagation for the RTE and DE.

The results of $\Phi$ and $J^{+}$calculated using the hybrid model

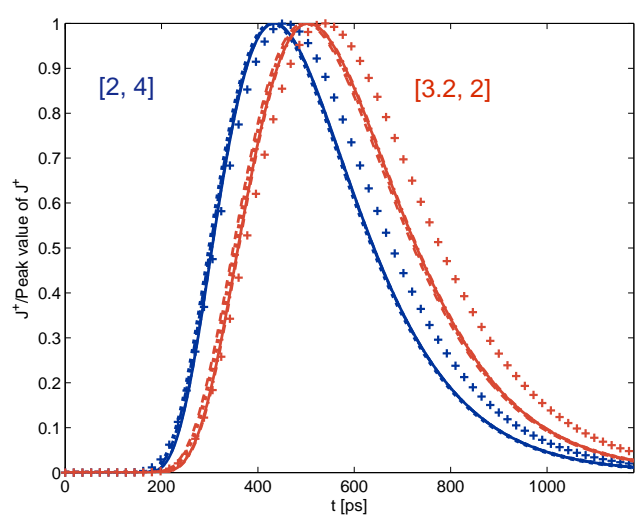

Figure 7: Calculated temporal profiles of $J^{+}$at detectors D5 $(\rho=2.8 \mathrm{~cm})$ and D6 $(\rho=3.2 \mathrm{~cm})$ on the surface of the medium with long source-detector distances. Crosses represent calculated results based on the DE, solid curves those based on the RTE with $g=0.8$ and the anisotropic source $\left(\Omega_{s x}, \Omega_{s y}\right)=(1,0)$, dashed curves those with $g=0.0$ and the anisotropic source $\left(\Omega_{s x}, \Omega_{s y}\right)=(1,0)$, dash-dot (chain) curves with $g=0.8$ and the isotropic source, and dotted curves those with $g=0.0$ and the isotropic source.

with $g=0.8$ and the anisotropic source $\left(\Omega_{s x}, \Omega_{s y}\right)=(1,0)$ evaluated as follows: although the hybrid model is constructed for different $g$ values and source conditions, the discussion can be generalized and is applicable to other conditions. Figure 11 plots the calculated results of $\Phi$ using the three models, the hybrid model (solid dots), the RTE (solid curves), and the DE (crosses) at two detectors inside the medium. The temporal profiles of $\Phi$ based on the hybrid model are consistent with those on the RTE at the positions near (detector D1) and far from the source (detector D2) within errors. Further, the temporal profiles calculated using the hybrid model connect without any gap at $t=t_{D A}$ even though the spatial hybrid model is changed as mentioned in sec 2.3 . This continuity indicates the validity of the proposed hybrid model. However, as shown in Fig. 11 (b), there are small differences between the RTE and the hybrid model in the later time region at detector D2. This difference results in a limitation of the current modification with the Robin boundary condition by only a simple adjustment of the $A$ value.

Time developments of $J^{+}$based on the three models on the boundaries are plotted in the Fig. 12. The calculated results for $\mathrm{J}^{+}$obtained with the hybrid model coincide with those of the RTE at the short and long distances (detectors D4 and D5). Modeling of light propagation at boundaries is more difficult than modeling inside media because the differences in the boundary conditions between the RTE and DE cause errors and discontinuities in the temporal profiles at $t=t_{D A}$. This suggests that the temporal hybrid does not hold at boundaries. To get around this, the RTE is solved in the source plane (the y-axis) even though $t$ is longer than $t_{D A}$ (see Fig. 2(b)), resulting in the continuous profiles of $\mathrm{J}^{+}$based on the hybrid model obtained in Fig. 12.

The proposed hybrid model can reduce computational load due to the spatial and temporal hybrids without loss in accuracy. Table 2 shows the computational times using the three models with time integration up to 1200 ps. The computational time 

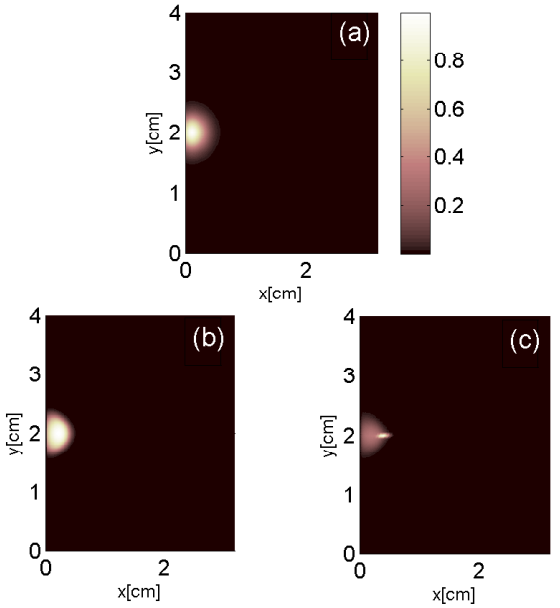

Figure 8: Spatial distributions of $\Phi$ normalized by its maximum value at a given time $t=20 \mathrm{ps}$ shorter than $t_{D A}$ based on (a) the DE, (b) the RTE with $g=0.8$ and the anisotropic source $\left(\Omega_{s x}, \Omega_{s y}\right)=(1,0)$, (c) the the RTE with $g=0.0$ and the anisotropic source $\left(\Omega_{s x}, \Omega_{s y}\right)=(1,0)$.
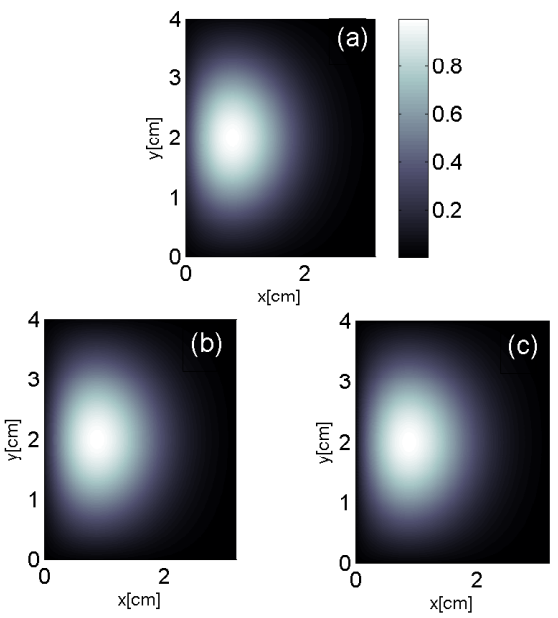

Figure 9: Spatial distributions of $\Phi$ normalized by its maximum value at at a given time $t=500 \mathrm{ps}$ longer than $t_{D A}$. Other details are the same as Fig. 8 .

using the hybrid model is reduced to a quarter of that using the RTE only.

\section{Conclusions}

For effective computation of light propagation in random media, we have constructed a space-time hybrid model based on the time-dependent RTE and DE by using the crossover length $\rho_{D A}$ and time $t_{D A}$. To determine $\rho_{D A}$ and $t_{D A}$, time developments of $\Phi$ and $J^{+}$obtained from the RTE and the DE in two dimensional homogeneous media under refractive-index mismatching were examined. The results show that the DA is valid at a space-time region, $\rho>\rho_{D A} \sim 10 / \mu_{t}^{\prime}$ and $t>t_{D A} \sim 20 / v \mu_{t}^{\prime}$, regardless of the random medium. The constructed hybrid model calculates light propagation adequately, and reduces computational load to a quarter compared with the full calculations of the RTE. In conclusion, the present model is potentially useful as a forward model in DOT.

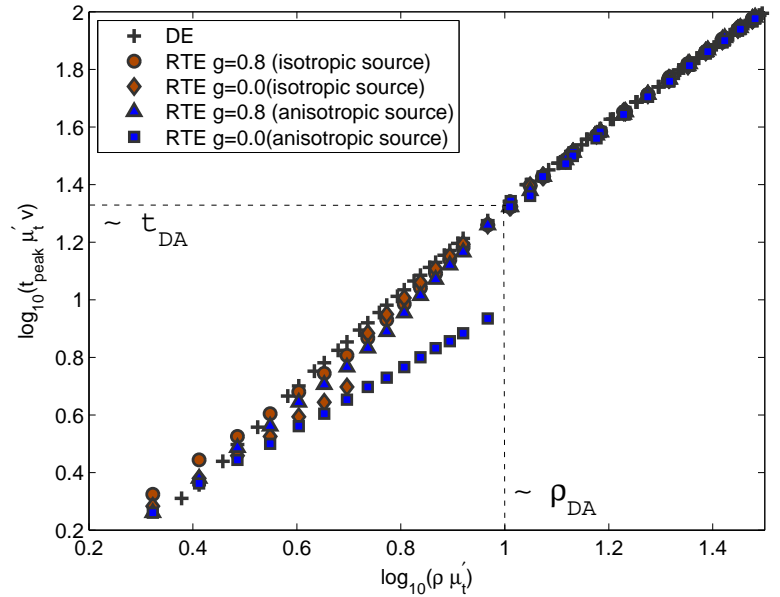

Figure 10: Peak times $t_{\text {peak }}$ of $\Phi$ calculated using RTE and DE as a function of the source-detector distance $\rho$. The $t_{\text {peak }}$ and $\rho$ values are normalized by $1 /\left(\mu_{t}^{\prime} v\right)$ and $1 / \mu_{t}^{\prime}$ respectively. Crosses represent $t_{\text {peak }} \mu_{t}^{\prime} v / t_{\text {ratio }}$ based on the DE. Solid circles and diamonds represent $t_{\text {peak }} \mu_{t}^{\prime} \nu$ based on the RTE with $g=0.8$ and with $g=0.0$ in the isotropic source, respectively. Solid triangles and squares are for $g=0.8$ and with $g=0.0$ in the anisotropic source, respectively.

\section{Acknowledgement(s)}

This work is supported by the Japan Science and Technology Agency. We wish to express appreciation to Mr. Tomoya Kosuge, Dr. Goro Nishimura, Dr. Eiji Okada, and Dr. Satoru Kohno for fruitful discussion.

\section{References}

[1] J. C. Hebden, S. R. Arridge, D. T. Delpy, Optical imaging in medicine : I . Experimental techniques, Phys. Med. Biol. 42 (1997) 825-840.

[2] Y. Hoshi, Functional near-infrared optical imaging : Utility and limitations in human brain mapping, Psychophysiology 40 (2003) 511-520.

[3] S. R. Arridge, Optical tomography in medical imaging, Inverse Prob. 15 (2) (1999) R41.

[4] A. D. Klose, U. Netz, J. Beuthan, A. H. Hielscher, Optical tomography using the time-independent equation of radiative transfer Part 1 : forward model, J. Quant. Spectrosc. Radiat. Transfer. 72 (2002) 691-713.

[5] A. D. Klose, A. H. Hielscher, Optical tomography using the timeindependent equation of radiative transfer Part 2 : inverse model, J. Quant. Spectrosc. Radiat. Transfer. 72 (2002) 715-732.

[6] G. S. Abdoulaev, A. H. Hielscher, Three-dimensional optical tomography with the equation of radiative transfer, J. Electron. Imaging. 12 (2003) 594-601.

[7] S. Okawa, Y. Hoshi, Y. Yamada, Improvement of image quality of time-domain diffuse optical tomography with lp sparsity regularization, Biomed. Opt. Express 2 (12) (2011) 3334-3348.

[8] A. H. Hielscher, R. E. Alcouffe, R. L. Barbour, Comparison of finitedifference transport and diffusion calculations for photon migration in homogeneous and heterogeneous tissues, Phys. Med. Biol. 43 (1998) 12851302.

[9] J. Heino, S. Arridge, J. Sikora, E. Somersalo, Anisotropic effects in highly scattering media, Phys. Rev. E 68 (2003) 031908 1-8.

[10] T. Tarvainen, M. Vauhkonen, V. Kolehmainen, J. Kaipio, Hybrid radiative-transfer-diffusion model for optical tomography, Appl. Opt. 44 (6) (2005) 876-886.

[11] T. Tarvainen, M. Vauhkonen, V. Kolehmainen, S. R. Arridge, J. P. Kaipio, Coupled radiative transfer equation and diffusion approximation model for photon migration in turbid medium with low-scattering and nonscattering regions, Phys Med Biol. 50 (2005) 4913-4930. 